\title{
Souffrance, compétence et résilience
}

Le cas des Témoins de Jéhovah

Suffering, competence and resilience: The Jehovah's Witnesses

\section{Régis Dericquebourg}

\section{(2) OpenEdition}

Journals

Édition électronique

URL : http://journals.openedition.org/span/696

DOI : 10.4000/span.696

ISSN : 2268-1558

Éditeur

École pratique des hautes études. Sciences humaines

Édition imprimée

Date de publication : 1 juin 2005

Pagination : $91-120$

ISSN : 0294-7080

Référence électronique

Régis Dericquebourg, «Souffrance, compétence et résilience », Systèmes de pensée en Afrique noire [En ligne], 17 | 2005, mis en ligne le 05 juin 2013, consulté le 19 avril 2019. URL : http:// journals.openedition.org/span/696 ; DOI : 10.4000/span.696 


\title{
Souffrance, compétence et résilience Le cas des Témoins de Jéhovah
}

\author{
Régis Dericquebourg \\ Maître de conférences à l'Universite \\ Charles de Gaulle (Lille III) \\ Groupe de sociologie des religions et de \\ la laïcité (EPHE/CNRS)
}

La réflexion développée ici se fonde sur ce qui est depuis longtemps notre champ de recherche : les groupes religieux minoritaires. Le cas examiné est celui des Témoins de Jéhovah, qui s'inscrit naturellement dans la problématique développée dans ce volume puisqu'ils insistent sur les souffrances endurées au cours de leur histoire. Nous ne nous en sommes cependant pas tenu à ce premier examen. En effet, le lien que les Témoins de Jéhovah font entre leurs souffrances et les compétences qu'elles leur auraient permis de gagner repose sur des présupposés implicites qu'il nous a paru intéressant de considérer en eux-mêmes. Pour cela, nous avons réalisé auprès d'échantillons d'étudiants une série de tests visant à mesurer jusqu'à quel point ces présupposés étaient partagés. Or à notre surprise, nous avons constaté que ces étudiants ne les partageaient pas, mais qu'ils en avaient d'autres, les amenant à faire un lien, d'une tout autre nature, entre souffrance et compétence.

\section{Exposé du problème}

La souffrance est d'abord un phénomène physique, émotionnel et cognitif individuel. Elle est aussi un phénomène social tant par la manière dont elle est considérée (fait biologique, punition divine) et traitée (médecine officielle, traitements psychologiques, invocations, remèdes de

L'excellence de la souffrance

Systèmes de pensée en Afrique noire, I7, 2005, pp. 91-I20 
1 Déjours, 1998 : 107.

119.

${ }^{2}$ Weber, $1996: 337$.

${ }^{3}$ Ibid. : 416. rebouteux, cure de medecine-men) que par la manière dont elle s'exprime (vocabulaire, catégories du langage) dans les différentes cultures et sous-cultures. Elle est sociale, dans la mesure où elle est parfois provoquée par des phénomènes collectifs tels que l'ostracisme, la discrimination, la sanction méritée ou injuste, par des rituels, par des contraintes que la société impose au corps ou à la conscience (telles que la formation d'une conscience morale stricte, cause de tourments et de culpabilité obsédante chez certains). La souffrance est sociale encore en ce qu'elle peut trouver son origine dans des formes de domination politiques (tortures...) et organisationnelles comme l'a montré Déjours ${ }^{1}$.

La souffrance produit des effets sociaux. De multiples organisations humanitaires ont été fondées pour informer le public de souffrances subies par des peuples ou des groupements humains, pour tenter d'y remédier, voire pour exiger des gouvernements qu'ils en éradiquent les causes. Enfin, socialement, la souffrance subie par un peuple ou un groupe appartient à la mémoire collective et modifie parfois les conduites à leur égard. Elle peut aussi provoquer des révoltes ou des révolutions.

Comme phénomène social, elle a suscité les réflexions des sociologues et, dans le domaine qui nous occupe, des sociologues des religions. Ainsi, pour Max Weber :

1) La souffrance a été considérée comme le symptôme de la haine divine et d'une culpabilité secrète (trouvant probablement sa cause dans une offense à Dieu). En ce cas, elle prend une valeur exclusivement négative (la faute), ce qui indique que l'homme souhaite que le bonheur qu'il atteint soit mérité et légitime (il n'a jamais commis de fautes); il revendique un droit au bonheur pour peu qu'il ne fasse rien pour subir la haine de Dieu. La religion y répond par une théodicée du bonheur ${ }^{2}$. Le dépassement de la souffrance devient une finalité de la religion de salut dans la mesure où elle propose une éthique permettant à l'homme d'écarter la souffrance ou de s'en libérer ${ }^{3}$.

2) Ce point de vue s'inverse avec la transfiguration religieuse de la souffrance. On passe de la souffrance collective d'un peuple puni à la prise en compte de la 
souffrance individuelle par la religion. Au plan magique, est apparue l'idée selon laquelle la souffrance provoquée par des automortifications conduit à l'acquisition de forces surhumaines de type magique. Des personnages peu ordinaires capables de délivrer l'homme de sa souffrance (les mystagogues) deviennent des recours ${ }^{4}$. En somme, la souffrance volontaire obtenue par l'automortification et les mortifications de l'abstinence et de la prière produit des états charismatiques.

3) La souffrance crée du lien social ${ }^{6}$ car des communautés se constituent sur la base d'une souffrance commune, celle causée par des persécutions actuelles ou conservées dans la mémoire collective et celle endurée par les martyrs.

4) La souffrance pose le problème du sens de la vie aux religions de salut. Les réponses apportées à l'existence de la souffrance sont historiquement différentes. En un premier temps ${ }^{7}$ des religions, le culte est avant tout communautaire. On intercède auprès des dieux pour obtenir des avantages collectifs : la pluie, la victoire sur l'ennemi, du gibier abondant. L'individu confie alors sa souffrance à des personnages en marge tels que les sorciers, les magiciens, les mystagogues, dont les succès assurent la notoriété du dieu qu'ils invoquent. Il se crée autour d'eux des groupements communautaires consacrés à la souffrance individuelle et à sa délivrance. La cure d'âme est née. Dans les religions, elle devient le privilège des prêtres. Ceux-ci peuvent déterminer les causes de la souffrance (lors de la confession des péchés) et recommander des conduites susceptibles de les éliminer. Un pas supplémentaire a été franchi lorsque s'est développée une religiosité du Sauveur qui présupposait un mythe libérateur c'est-à-dire une conception du monde où la souffrance est centrale. Chez les Juifs, la souffrance d'un peuple fut d'abord placée au centre des espérances religieuses de délivrance. Toutefois, le Sauveur ne pouvait garantir le salut de chaque individu qui s'adressait à lui. Alors, presque toujours, les espérances de salut ont donné naissance à quelques formes de "théodicée de la souffrance " où cette dernière devenait positive, ce qu'elle n'était pas - on l'a dit plus haut - à l'origine ${ }^{8}$. Il s'agit d'expliquer la souffrance

\author{
${ }^{4}$ Ibid. : 338. \\ ${ }^{5}$ Ibid. : 342. \\ ${ }^{6}$ Ibid. : 419. \\ ${ }^{7}$ Ceci n'implique pas \\ que Max Weber ait une \\ conception évolution. \\ niste de l'histoire. \\ ${ }^{8}$ Weber, $1996: 341$.
}


${ }^{9}$ Cf. Nowak, 1972.

${ }^{10}$ Weber, $2003: 355$.

${ }^{11}$ Association de Défense des Familles et de I'Individu; Centre de documentation, d'éducation et d'action Contre les Manipula. tions Mentales. injuste et imméritée comme Jean Chrysostome l'a fait'. Ceci n'empêche pas le recours à des voies annexes de cessation de la souffrance : quand les promesses du Sauveur ou du prophète ne répondaient pas suffisamment aux besoins de personnes socialement dévalorisées, une religiosité secondaire de salut s'est développée à l'ombre de la doctrine officielle (dévotions populaires?).

5) Par ailleurs, dans Hindouisme et bouddhisme, Max Weber relève un autre mode de rapport religieux à la souffrance. Ces religions proposent des ascèses qui procurent une nouvelle compréhension du monde, via l'illumination, où l'on s'affranchit définitivement de la souffrance ${ }^{10}$.

Dans ce relevé des considérations wébériennes sur la souffrance, deux idées-force apparaissent : La souffrance est créatrice de lien social et la souffrance peut produire des états charismatiques, c'est-à-dire des états reconnus comme non ordinaires par une communauté d'individus, producteurs d'un don manifestant une virtuosité particulière. Les religiosités asiatiques procèdent d'une manière inverse puisque le virtuose est celui qui réussit dans la voie de l'extinction de la souffrance en atteignant l'illumination. Dans les deux cas, la souffrance produit une virtuosité religieuse qui est une compétence particulière. Mais peut-on aller plus loin et se demander si des persécutions subies peuvent constituer un critère de compétence chez un groupe religieux? Nous pensons aux Témoins de Jéhovah, qui rappellent les souffrances qu'ils ont endurées au moment où ils sont controversés dans la société française.

\section{Les Témoins de Jéhovah : souffrance et volonté de reconnaissance religieuse}

Les Témoins de Jéhovah ont toujours présenté dans leurs ouvrages les persécutions subies, mais depuis une dizaine d'années, ils les évoquent plus intensément. En France surtout, car depuis la montée en puissance de la lutte antisecte, d'abord associative (ADFI, $\mathrm{CCMM}^{11} \ldots$ ) puis relayée au plan gouvernemental (Rapport Vivien, Mission interministérielle de lutte contre les sectes, loi About- 
Picard), ils ont été mis en cause à propos de l'éducation de leurs enfants, à propos du refus des transfusions sanguines, à propos de leur insoumission à l'armée ${ }^{12}$. Un redressement fiscal très lourd leur a été signifié ${ }^{13} \mathrm{car}$, n'ayant pas la pleine reconnaissance comme association cultuelle, ils ne peuvent pas bénéficier de la capacité à recevoir des dons et des legs (loi de 1905-1907). Par son importance, cette sanction fiscale pourrait mettre en cause leur présence dans notre pays. L'association cultuelle des Témoins de Jéhovah a réagi sur deux plans : un combat juridique pour bénéficier des mêmes avantages que les Églises établies ${ }^{14}$ et un appel à l'opinion publique pour contrer les affres subies au plan institutionnel mais aussi au plan individuel car des fidèles ont été inquiétés dans leur vie professionnelle ou privée en raison d'une appartenance religieuse devenue controversée.

Au plan juridique, ils ont demandé le statut de culte reconnu. Pour cela, ils ont exigé les bénéfices que procure celui-ci. Ils ont refusé de payer les taxes foncières dont sont exonérées les grandes confessions, ce qu'ils n'avaient jamais fait jusque-là puisque leur statut légal leur importait peu. Ils ont plaidé leur cause avec succès devant les tribunaux administratifs et devant le Conseil d'État. Toutefois, le bureau des cultes n'en n'a pas tiré toutes les conséquences, à savoir leur accorder le statut plénier de culte reconnu. Comme l'association cultuelle des Témoins de Jéhovah a toujours réussi à exister sans exonération fiscale, on peut se demander si ce qu'ils recherchent en revendiquant ce statut n'est pas en fait une reconnaissance comme "véritable religion". Le statut que le bureau des cultes est susceptible d'accorder n'a pas en principe une telle valeur puisque l'État se prononce sans se préoccuper de la valeur des croyances, mais il a bel et bien une valeur symbolique puisqu'il permet à l'association qui l'obtient d'intégrer le cercle très fermé où figurent déjà les grandes confessions. Ce statut n'est pas négligeable, comme on l'a vu dans l'affaire d'une Église Évangélique non reconnue, pour laquelle l'État a demandé l'avis de la Fédération protestante de France, interlocuteur reconnu.

\author{
12 Réglée depuis par la \\ circulaire Léotard et la \\ fin de la conscription. \\ 13 L'affaire n'est pas \\ réglée ce jour. \\ ${ }^{14}$ En France, toute \\ association à caractère \\ spirituel ou religieux \\ peut se proclamer \\ " association cultuelle » \\ (loi de 1905). Toutefois, \\ cela ne l'autorise pas \\ à obtenir certains \\ avantages dont \\ disposent les grandes \\ confessions (catholi- \\ cisme, protestantisme, \\ judaïsme, islam) à \\ savoir : aumônerie de \\ prisons, d'établisse. \\ ments secondaires et \\ de l'armée, possibilité \\ de recevoir des dons et \\ legs, exonérations des \\ taxes foncières sur les \\ édifices cultuels. II faut \\ pour cela obtenir une \\ « reconnaissance » en \\ association cultuelle \\ plénière donnée par le \\ bureau des cultes du \\ Ministère de l'Intérieur \\ (loi de 1907). Comme \\ l'ont fait remarquer \\ le professeur Jacques \\ Robert et le doyen \\ Carbonnier dans leurs \\ études sur la loi de \\ 1905 , tous les cultes \\ n'ont pas droit au \\ même traitement. On \\ trouve d'une certaine \\ manière l'introduction \\ implicite d'un régime \\ des cultes reconnus.
}


${ }^{15}$ Canonici, 1998.

${ }^{16}$ Zuber et Baubérot, 2000.

${ }^{17}$ Les Témoins de Jéhovah, 1993.

18 Les Témoins de Jéhovah, 1971.
Au plan public, l'élément majeur de l'action des Témoins de Jéhovah a consisté à rappeler leurs souffrances passées. Ils ont fait circuler deux cassettes vidéo: Les triangles violets (1996) et La fermeté des Témoins de Jéhovah face à la persécution nazie (1997). Dans cette dernière, dix historiens européens et américains évoquent les souffrances des Témoins sous le troisième Reich à côté d'une vingtaine de survivants des camps de concentration. Par ailleurs, deux ouvrages ont été consacrés à leur période noire: The Spirit and the Sword (texte et images d'une conférence donnée le 29 septembre 1994 au Musée de l'Holocauste à Washington), et Les Témoins de Jéhovah face à Hitler ${ }^{15}$. Enfin, le Cercle européen des Témoins de Jéhovah anciens déportés et internés a organisé en France une exposition itinérante sur la déportation des fidèles jéhovistes pendant la seconde guerre mondiale.

Ainsi, au moment où ils sont controversés, les Témoins de Jéhovah rappellent avec plus de force les persécutions subies. De cette manière, ils s'inscrivent dans la lignée des groupes martyrisés (premiers Chrétiens, Juifs, Protestants $\left.{ }^{16}\right)$. La même démarche a été faite par les homosexuels au moment de "la sortie du placard", c'est-à-dire quand ils ont commencé à se donner une visibilité et à revendiquer la même considération que les hétérosexuels. Cette époque a été marquée par la publication d'ouvrages décrivant l'ostracisation des homosexuels dans l'histoire et en particulier le sort des "triangles roses " pendant la seconde guerre mondiale.

Les Témoins ne s'arrêtent pas aux affres de la seconde guerre mondiale. Le chapitre 29 de leur historique ${ }^{17}$ est une mise en perspective de toutes les autres persécutions subies, qu'on peut lire comme un récit des souffrances endurées à cause de leur foi. Certes, ils l'avaient déjà fait précédemment ${ }^{18}$ mais le récit de leurs vicissitudes était moins étoffé. Toutefois, on y lisait déjà que le Christ était le premier Témoin de Jéhovah (généalogie revendiquée) et que le Jéhovisme endurait depuis ses débuts la persécution des premiers Chrétiens. Dans l'interprétation que les Témoins donnent de leur souffrance, celle-ci apparaît comme : 
- sublimée par la lutte contre le totalitarisme et pour les libertés religieuses. Ils illustrent cet argument par une citation de l'historienne Christine King ${ }^{19}$ : "Les témoins de Jéhovah lançaient un défi au concept totalitaire de cette nouvelle société, et ce défi, qui s'affirmait de jour en jour, dérangeait les architectes de l'ordre nouveau. " La thèse des Témoins de Jéhovah défenseurs des libertés fondamentales a également été développée par Gary Botting ${ }^{20}$ et par James Penton $^{21}$, tous deux universitaires jéhovistes au moment de la parution de leurs ouvrages;

- voulue par Dieu. Pour les Témoins de Jéhovah, la Watchtower Society ${ }^{22}$ est le canal prévu et utilisé par Dieu pour répandre la vérité dans le monde. Ils affirment que «le Seigneur peut susciter des Témoins dans n'importe quel pays pour tenir bien haut l'étendard de la vérité et faire connaître le nom de Jéhovah " $^{23}$. Le "n'importe quel pays" renvoie aux nations qui les ont persécutés;

- transcendée par une annonce prophétique. Les souffrances qu'ils ont endurées ne sont pas sociologiquement interprétées en termes d'hostilité envers les non-conformismes religieux, envers des pratiques sociales désapprouvées, envers une protestation socio-religieuse. Pour eux, elles ont été prophétisées par Jésus. Nous lisons dans leur historique qu'au cours de la dernière soirée que Jésus a passée avec ses apôtres avant de mourir, il leur a rappelé ceci : «Un esclave n'est pas plus grand que son maître. S'ils m'ont persécuté, ils vous persécuteront aussi. S'ils ont observé ma parole, ils observeront aussi la vôtre. Mais ils feront tout cela à cause de mon nom " (Matthieu 24, 9) 24 . Ainsi la souffrance des Témoins de Jéhovah est transcendée par une annonce prophétique et elle reçoit une plus-value d'autorité dans la mesure où elle est identifiée à la souffrance du Christ;

- légitimée par une cosmogonie et une éthique. Les Témoins se disent persécutés parce qu'ils représentent une partie du cosmos : celle qui est sous l'emprise de Dieu et donc du Bien, haïe par la partie du monde sous l'emprise de Satan et donc du Mal. Ils affirment: "Depuis longtemps, les publications de la Société Watchtower montrent que le premier livre de la Bible prédit dans un langage

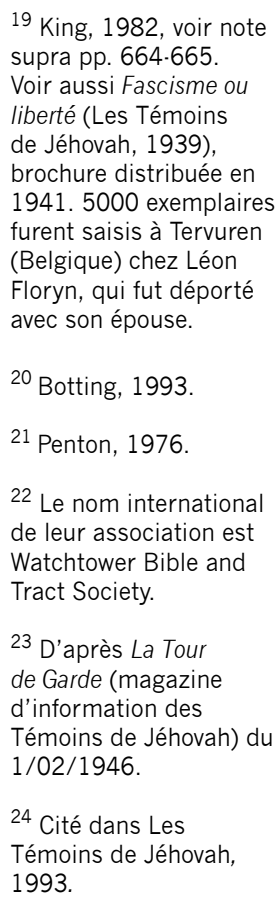


${ }^{25}$ Ibid. : 676.

26 Ibid. : 450.

27 Ibid. : 662.

28 Ibid. : 662.

${ }^{29} \mathrm{King}$, cité Ibid. : 664.665. symbolique l'inimitié ou la haine que Satan, le Diable et ceux qu'il domine éprouvent à l'égard de l'organisation céleste de Jéhovah et de ses représentants sur la terre " (Gen. 3, 15; Jean 8, 33-44; Révél. 12, 9-17), à quoi ils ajoutent : " Il n'existe que deux organisations principales : celle de Jéhovah et celle de Satan $»^{25}$;

- supportée avec une force peu commune. Les Témoins insistent sur le fait que leurs croyances les ont aidés à surmonter leurs souffrances, en ces termes par exemple : «Si les Témoins ont pu faire preuve de courage et de persévérance, c'est notamment parce qu'ils étaient bien nourris spirituellement ${ }^{26}$. Ils rappellent que dans les camps nazis, ils pouvaient être libérés sur le champ s'ils signaient un acte de renonciation. Pour les Témoins, le faible nombre d'apostasies exprime une acceptation volontaire de la souffrance au profit de la vérité car ils s'identifient aux anges de Dieu : «La Bible dit que les anges de Dieu joueraient un rôle capital dans ce domaine. C'est pourquoi la bonne nouvelle du royaume se propagerait dans le monde comme une sonnerie de trompette d'origine supra-humaine $"^{27}$. Pour eux, le fait que « dès 1935, leur message ait touché 149 pays au Nord et au Sud, à l'Est et à l'Ouest, d'une extrémité de la terre à l'autre ${ }^{28}$ en constitue la preuve. Il n'est donc pas étonnant qu'ils aient intitulé «Fermeté " une de leurs cassettes opposant leur "ferme conviction" à celle des nazis. En ce sens, ils citent à l'appui de leur thèse la phrase de Christine King : «les Témoins étaient assurés de la fidélité totale et inflexible de leurs membres, et ce jusqu'à la mort " ${ }^{29}$.

- socialement reconnue. Les Témoins ne manquent pas de citer des témoignages reconnaissant leur caractère peu commun, comme la lettre de Geneviève De Gaulle (nièce du général De Gaulle) parlant de sa déportation au camp de Ravensbrück où l'on comptait 500 Témoins de Jéhovah féminines: "J'ai pour elles une véritable admiration. Elles appartenaient à différentes nationalités : allemandes, polonaises, russes ou tchèques et ont subi pour leurs croyances de très grandes souffrances [...]. Toutes faisaient preuve d'un très grand courage et finissaient par en imposer aux S.S. eux-mêmes. Elles auraient pu être libres 
sur le champ si elles avaient renoncé à leur foi. Au contraire, elles ne cessaient de résister, réussissant à introduire dans le camp des livres et des tracts $"{ }^{30}$. Une lettre du même type a été écrite par Léon Blum. Le courage et la conviction des Témoins ont été remarqués par l'un de leurs geôliers, Rudolf Hoess, commandant d'Auschwitz, qui a témoigné d'exécutions de Témoins de Jéhovah refusant d'abjurer leur foi. Il écrit : "C'est ainsi que je me représentais les premiers martyrs du christianisme, debout dans l'arène en attendant d'être dévorés par les bêtes fauves, avec une expression de joie extatique, les yeux levés au ciel, les mains jointes pour la prière, ces hommes accueillirent la mort. Tous ceux qui avaient assisté à l'exécution, même les soldats du peloton, étaient profondément émus ${ }^{31}$.

- valorisée par la condition modeste des Témoins de Jéhovah. Ces derniers affirment que, comme Celse, philosophe romain du II ${ }^{\mathrm{e}}$ siècle qui ironisait sur le fait que des gens modestes soient prédicateurs de l'Evangile, on reproche à présent aux Témoins d'être des gens ordinaires dont la position sociale n'en impose à personne ${ }^{32}$. En ceci, les Témoins s'identifient aux premiers chrétiens et aux apôtres de Jésus « eux-mêmes issus de la classe ouvrière [sic] » et ils subliment leurs souffrances en en faisant celles des couches modestes de la population espérant une nouvelle dispensation.

- surmontée et triomphante. Le récit des vicissitudes subies par les Témoins de Jéhovah s'achève par la conclusion que ces derniers ont triomphé de leurs persécuteurs. La souffrance endurée a été la source de leur victoire : « La persécution brutale et les connaissances de la guerre totale n'ont pas pu empêcher que, comme prédit, des gens se soient rassemblés dans une grande maison spirituelle de Jéhovah pour l'adorer (Isaïe, 2, 2-4).» Les dictateurs, les régimes politiques qui les ont fait souffrir ont disparu. Le clergé en qui ils trouvent le moteur de leur persécution voit son audience faiblir. Ils en concluent qu' « il est évident qu'il n'appartient à aucun homme, ni à aucun gouvernement d'autoriser la pratique du culte pur $»^{33}$.

30 Ibid. : 664.

${ }^{31}$ King, cité Ibid. : 663.

32 Ibid. : 548.

33 Ibid. : 696. un destin de souffrance prophétisé et victorieusement sur- 
monté grâce à une force peu ordinaire d' " homme de Dieu ". En reprenant la notion de type d'homme (Menschentyp) qui, chez Weber, désigne les figures sociales de l'humanité (comme l'homme du gain) que les structures sociales façonnent, les Témoins apparaîtraient dans une histoire revisitée par le judéo-christianisme comme des " hommes de la souffrance ", celle-ci étant sans cesse renouvelée et surmontée.

En retournant à notre problème, on peut faire l'hypothèse que les Témoins de Jéhovah rappellent leurs affres et leur victoire sur les persécuteurs pour obtenir la même légitimité et la même reconnaissance que les premiers chrétiens, lesquels ont perduré dans leur foi en dépit de leurs persécutions et ont engendré des Églises dont on ne conteste pas le caractère religieux. Encore faut-il savoir ce qu'est une compétence.

\section{La notion de compétence}

Pour Claude Levy-Leboyer, « compétence » est un mot qui s'est répandu récemment dans le monde du travai ${ }^{34}$. Aussi est-ce du côté de la psychologie du travail et des ressources humaines que l'on peut en chercher la définition. L'auteur insiste sur la difficulté à décrire la compétence d'un salarié et les compétences en général. Ceci provient de la multiplicité des références, tantôt liées à une tâche, tantôt à une activité donnée ou encore à un ensemble d'activités, comme c'est le cas pour la compétence linguistique ou la compétence d'encadrement. Il l'illustre en montrant que les listes de critères de compétence d'un cadre établies par divers auteurs forment des ensembles disparates. Par ailleurs, l'acquisition de compétences mobilise des compétences différentes. Lévy-Leboyer propose sa propre définition : les compétences «constituent un lien entre d'une part, les missions à accomplir et les comportements mis en jeu pour ce faire et, d'autre part, les qualités individuelles nécessaires pour se comporter d'une manière satisfaisante $»^{35}$. Cette définition des compétences (et non de la

${ }^{34}$ Levy-Leboyer, 2004

35 Ibid. : 35 compétence) prend en compte des facteurs dispositionnels et des conduites évaluées par la satisfaction du donneur 
d'ordres. D'une manière générale, on constate un glissement de la compétence vers les compétences.

Pour David Courpasson et Yves Frédéric Livian ${ }^{36}$, la notion de compétence, dont l'utilisation est abusive, déconstruit les métiers pour aboutir à des ensembles professionnels diaphanes et inconsistants (comme les « personnels de contact ", le " personnel du management »). Ces derniers sont des catégorisations sociales artificielles reposant plus sur la notion d'individualisme que sur la notion de groupes professionnels qualifiés et reconnus sur la base d'un savoir ou d'une technique acquis, et qui vont à l'encontre de la revendication d'une définition claire du métier chez les salariés. L'ergonome Maurice de Montmollin affirme que les compétences professionnelles sont des "ensembles stabilisés de savoirs et de savoir-faire, de conduites types, de procédures standard, de types de raisonnement que l'on peut mettre en œuvre sans apprentissages nouveaux. Les compétences structurent les acquis de l'histoire personnelle, elles permettent l'anticipation des phénomènes, l'implicite dans les instructions, la variabilité dans la tâche. Les compétences, cela permet ainsi de savoir à qui l'on a affaire, sur qui on peut compter pour accomplir telle tâche. On prend ainsi des risques car personne n'est jamais complètement fiable... $»^{37}$. Globalement, l'auteur émet des réserves sur cette notion car le salarié est un " opérateur humain » dont le savoir-faire se rapporte à une tâche précise, à un poste de travail, à une rémunération qui l'identifient dans l'entreprise (signification sociale de la compétence). Dans un autre $\operatorname{article}^{38}$, l'auteur accentue sa réserve. Il refuse la notion de « compétences génériques" au profit des connaissances et du savoir-faire généré par le salarié selon les situations. Il illustre son point de vue en prenant l'exemple du pilote confronté à une difficulté en vol. La personnalité est exclue du champ de l'évaluation des compétences. En somme, il y aurait des compétences pour mais pas de compétence en général.

Au cours de notre réflexion, nous nous sommes demandé si la souffrance pouvait être prise en compte dans l'évaluation d'une compétence. Pour cela, nous avons réa-

\footnotetext{
${ }^{36}$ Courpasson et Livian, 1991.

37 Montmollin, 2001 : $11 \cdot 12$.

38 Montmollin, 1993 : 205.210.
} 
39 Weiner et Kukla, 1970. Voir aussi Desrumaux, 1996 lisé une étude auprès d'étudiants en psychologie et, parmi eux, auprès d'une promotion du diplôme d'études spécialisées en psychologie du travail. Ces étudiants reçoivent un enseignement sur l'évaluation des compétences et certains la pratiquent déjà pendant les stages ou dans leur emploi. L'étude que nous avons menée (annexe 2) montre que les étudiants en psychologie rejettent l'idée selon laquelle "se donner du mal (souffrance dans l'emploi) sur un poste de travail est un critère de compétence. En revanche, l'appel à l'effort est très important dans l'acquisition de la compétence scolaire, c'est-à-dire dans l'accès à un niveau de connaissance requis et objectivable. Nous le montrons dans un échantillon de bulletins trimestriels de collégiens (annexe 1). Or, nous avons constaté empiriquement que l'effort est lié à une souffrance (annexe 3). Autrement dit, dans l'apprentissage scolaire, qui équivaut à l'acquisition de compétences, et peut-être dans les autres apprentissages, on valorise une forme de souffrance-effort. Ceci est en accord avec l'évaluation de l'item d'un autre questionnaire (annexe 6) administré aux étudiants : "Un travail qui a été fait dans l'effort doit être mieux apprécié subjectivement qu'un travail fait avec facilité même si les deux aboutissent à la même note " (le mot « note " laissait entendre qu'il s'agissait d'un travail scolaire). L'accord avec la proposition est positif de manière statistiquement significative. Ceci rejoint les résultat d'une expérience de Weiner et Kukla où 20 étudiants devaient évaluer des élèves. Les élèves motivés à l'effort, aptes ou non aptes, sont plus récompensés que les élèves non motivés à l'effort ${ }^{39}$, qu'ils soient aptes ou non aptes. En revanche, la proposition : "L'acquisition de compétences s'accompagne toujours d'une certaines souffrance " ne reçoit qu'une évaluation moyenne. Le mot souffrance a sans doute été jugé excessif par les étudiants. Au plan du groupe, la formule : "Les groupes qui ont souffert ont acquis une compétence dans la vie » reçoit une note significativement supérieure à la moyenne. Nous concluons qu'il existe un lien entre l'effort (associé à la souffrance) et l'acquisition de compétences à l'exception de certaines situations comme le travail salarié. 


\section{La compétence en religion}

Dans les groupes religieux, divers acteurs sociaux peuvent être définis par une compétence. Celle des clercs consiste à administrer la grâce à travers un système sacramentel dont leur Église est dépositaire et à faire la "cure d'âme ". S'y ajoutent des activités plus temporelles telles que l'administration et l'animation d'une assemblée de fidèles. De nombreuses études sont consacrées aux activités des ecclésiastiques $^{40}$. On a même tenté de faire des études de type ergonomique sur la base de la méthode des budgets-temps. Toutefois, Thomas Gannon ${ }^{41}$ fait remarquer que le clergé ne dispose pas d'un savoir-faire technique applicable à la solution des problèmes empiriques : il interprète les événements mais ne résout pas les problèmes. On peut ajouter que le charisme institutionnel dont il est le transmetteur lui est donné et n'est pas éveillé par une ascèse particulière, surtout douloureuse. Les théologiens officiels des Églises auxquels on attribue une compétence pour interpréter les textes sacrés ne doivent celle-ci qu'à l'étude et non à quelque mortification. Seuls les virtuoses qui atteignent l'extase et éveillent des dons charismatiques à l'aide de mortifications acquièrent une compétence exceptionnelle grâce à la souffrance. Dans les sectes, il n'existe pas de qualifications particulières puisque ces mouvements de laïcs n'acceptent pas la notion de clergé. Les fidèles peuvent prendre des charges, avec l'accord de l'assemblée. Toutefois, on trouve parfois une spécialisation des fidèles pour occuper des tâches.

Dans le jéhovisme, le Collège central de Brooklyn, qui élabore la doctrine en interprétant les Écritures, rédige les écrits diffusés par le mouvement et administre la Watchtower Society, constitue un ordre-curie de permanents qui coiffe l'organisation alors que dans la congrégation locale, la plupart des rôles pris par les «serviteurs " sont tournants. Naturellement, il peut s'opérer des glissements. Les individus peuvent être choisis par l'assemblée ou cooptés par les dirigeants en place sur la base d'une fidélité dans l'engagement religieux, d'une confiance accordée ou d'une «sagesse " attribuée. Max Weber évoque ceci à propos des

\author{
40 On en trouve déjà \\ beaucoup de références \\ dans Prêtres, pasteurs et \\ rabbins dans la société \\ contemporaine, 1982 , \\ notamment dans la \\ contribution de Jean \\ Séguy. Voir aussi Jean- \\ Paul Willaime, 1989. \\ ${ }^{41}$ Gannon, 1971.
}


42 Sur cette question des charges et du charisme de l'état de grâce, voir Max Weber, $1964: 283 \cdot 286$ sectes protestantes où ni un charisme de fonction impersonnel, ni une formation théologique (quand elle existe, elle est une simple condition technique préalable et son absence ne peut être invoquée à l'encontre du candidat à une charge) ne sont requis pour diriger l'assemblée. La compétence serait dans ce cas un "charisme de l'état de grâce ", succédané du charisme de fonction, reconnu par les fidèles, et qui a parfois donné lieu à des " certificats de qualification religieuse " attribués sur la base d'enquêtes. Il n'empêche que pour l'auteur, un glissement peut s'opérer en direction d'une charge institutionnelle comme dans le méthodisme où la différence entre les prédicateurs ordonnés et les fidèles a été abolie puis réintroduite par la création en 1836 d'une ordination formelle permettant de donner les sacrements. Ceci a finalement abouti à la formation d'un nouveau clergét ${ }^{2}$. Mais en aucun cas, la souffrance n'intervient dans l'éveil du charisme de l'état de grâce.

Au plan collectif, il est encore plus difficile de définir la compétence d'un groupe religieux car celle-ci renvoie à un modèle idéal de compétence religieuse. Néanmoins, nous avons tenté de trouver des critères de compétence religieuse auprès d'un échantillon d'étudiants pour voir si la souffrance du groupe y participait.

\section{Critères empiriques d'une compétence religieuse}

Nous avons demandé à un échantillon de 101 étudiants en psychologie de choisir dans une liste de propositions celles qui pourraient caractériser une vraie religion (expression qui selon nous opérationalise la compétence religieuse dans le contexte de la polémique actuelle qui oppose les fausses religions aux vraies religions). Nous avons tenté de traduire la compétence en critères objectifs, c'est-àdire en termes de conduites et non en termes d'idéologie ou de doctrine (annexe 4).

Les résultats sont $a$ priori surprenants car ils s'éloignent de l'image classique d'une religion. En effet, le premier critère définitoire d'une vraie religion serait de laisser le fidèle 
libre de ne pas croire en Dieu. Les deux items qui viennent ensuite ex-aequo (assez loin devant " défend les droits de l'homme ") sont "donner un sens à la vie " et "permet à ses fidèles de surmonter les épreuves de la vie ». La place accordée au premier de ces deux items rejoint l'enquête d'Antoine Delestre ${ }^{43}$ montrant l'importance de la quête de sens chez les étudiants. Quant au second item, il marque l'importance accordée à une compétence qui sera examinée plus loin : la résilience. L'image de la vraie religion est plutôt le bouddhisme et certains groupes du Nouvel Âge. Naturellement, cette représentation est limitée à notre échantillon mais on peut admettre que des tendances aussi fortes se reflèteraient dans un public plus large.

\section{Discussion}

Un lien existe entre la souffrance et la compétence mais il n'apparaît pas stable. On le trouve dans les situations d'apprentissage. Au plan collectif, les étudiants pensent que les groupes qui ont souffert ont acquis une compétence particulière mais ils évaluent négativement et de manière significative la proposition selon laquelle un groupe de croyants ne devient une vraie religion que s'il a été persécuté dans ses débuts. Nous n'évoquerons pas les propositions concernant la compétence de groupes particuliers car les compétences soumises dans le questionnaire étaient certainement inadéquates. En revanche, la capacité qu'une religion donne à ses fidèles de surmonter les épreuves de la vie (manière euphémisée de nommer les souffrances de l'existence) est considérée chez les étudiants comme le critère d'une vraie religion et de la validité des croyances. Une des compétences religieuses serait d'éveiller chez les fidèles une capacité à surmonter la souffrance. En insistant sur le fait qu'ils triomphent de la persécution et de l'adversité, les Témoins de Jéhovah montreraient que leur socialisation religieuse comporte un facteur de résilience.

La résilience est tantôt définie comme « la capacité de sortir vainqueur d'une épreuve qui aurait pu être traumatique avec une force renouvelée - la résilience impliquant 
${ }^{44}$ Arnaud, $2003: 7$.

${ }^{45}$ Patterson et al.

(1994) cité par Detraux, $2002: 29 \cdot 40$

46 Ibid. : 32.

${ }^{47}$ Pelchat, Lefebvre et Damiani, $2002: 46$.

48 Voir par exemple Cyrulnik, 1999.

${ }^{49}$ Houssier, 2002 : 69. l'adaptation face au danger, le développement normal en dépit des risques et le ressaisissement de soi après un traumatisme " ${ }^{44}-$, tantôt comme l'aptitude à fonctionner de façon adaptée et à devenir compétent lorsque des événements de vie stressants se présentent ${ }^{45}$. Dans ce cas, elle est considérée comme la capacité de continuer à se développer et à augmenter ses compétences dans l'adversité. Pour J.J. Detraux, la résilience est liée à l'acquisition de compétences individuelles qui permettent de prendre le contrôle, de piloter des actions, de comprendre ce qui nous arrive et de donner un contenant à un contenu douloureux, pénible. La notion de sens donné aux événements est déterminante chez cet auteur ${ }^{46}$. Diane Pelchat, Hélène Lefebvre, Carole Damiani ${ }^{47}$ font la même constatation pour le groupe familial lorsqu'il est confronté à une situation douloureuse telle que la mort d'un enfant. La famille qui « fait face " se transforme en donnant un sens positif à l'événement au sein d'un processus de réadaptation. Boris Cyrulnik évoque d'autres facteurs de résilience comme la sublimation, l'altruisme, l'humour, la rencontre avec des substituts affectifs ${ }^{48}$.

Bruno Bettelheim utilisa un autre facteur de résilience. La transmission de son expérience et le témoignage devinrent le fil directeur de sa vie depuis le moment de sa libération du camp de Buchenwald jusqu'à sa mort ${ }^{49}$. Globalement, les chercheurs s'accordent sur le fait qu'on ne peut être résilient tout seul. Ils supposent que les parents, les groupes sociaux de base enseignent à mobiliser des ressources, la résilience étant elle-même selon Boris Cyrulnik un apprentissage de la vie, l'éveil de nouvelles compétences. A propos des Témoins de Jéhovah, Bruno Bettelheim avait fait la constatation suivante : "Non seulement, ils faisaient preuve d'une dignité et d'un comportement moral exceptionnels mais ils semblaient protégés contre l'influence du milieu concentrationnaire qui détruisait rapidement les personnes que mes amis psychanalystes et moi-même jugions bien intégrées..." Il ajoute: "Les témoins de Jéhovah étaient détenus en tant qu'objecteurs de conscience. Ils étaient encore moins affectés par leur détention et conservaient leur intégrité grâce à des convictions religieuses 
rigides. Leur seul crime aux yeux des S.S. étant de refuser de porter les armes, on leur offrait fréquemment de les libérer s'ils accomplissaient leur service militaire. Ils refusaient toujours $"^{50}$. De la même façon, Jorge Semprun, étonné de leur résistance à la souffrance dans les camps, écrit : « Silencieux, dévoués et inusables, ils attendaient patiemment la fin des maux apocalyptiques qu'avait provoqué la chute de Satan sur la terre, en 1914, et le millenium qui s'ensuivait, à une date prochaine, encore qu'indéterminée, ouvrant les portes d'un Monde Nouveau où les élus gouvernaient depuis leur demeure céleste $~^{51}$.

Revenons maintenant sur les deux volets de cet article. Les Témoins de Jéhovah détaillent dans leurs publications leurs souffrances passées et actuelles, entendant montrer qu'ils constituent une vraie religion, puisqu'ils ont connu le même sort que les premiers chrétiens ou les autres groupes persécutés. Autrement dit, ils présentent leurs souffrances comme la marque d'une compétence. Or cette vue ne rencontre pas d'écho dans notre échantillon d'étudiants, lesquels n'établissent pas de lien entre souffrance et compétence. Par contre, ceux-ci considèrent que l'aptitude à surmonter la souffrance est une authentique compétence. Il y a un autre point de désaccord entre les présuppositions implicites des Témoins de Jéhovah et les étudiants de notre échantillon : pour ceux-ci, la reconnaissance comme « culte reconnu de plein exercice " ne porte pas à une évaluation favorable. Une telle reconnaissance règlerait peut-être la situation des Témoins de Jéhovah vis-à-vis de l'administration fiscale, mais elle n'améliorerait pas nécessairement leur image dans l'opinion. En revanche, ils ont des chances d'être entendus sur le point de la souffrance surmontée.

Exister encore après tant de persécutions et être plus fort que jamais serait un élément favorable puisque notre échantillon considère la capacité à mobiliser des ressources pour surmonter la souffrance comme une compétence religieuse. En un mot, les deux volets de cette étude font apparaître la prégnance d'une image de la religion-résilience. C'est certainement là un trait qui justifierait des recherches ultérieures.

\footnotetext{
50 Bettheleim, 1972.

${ }^{51}$ Canonici, 1998 : 306.
} 


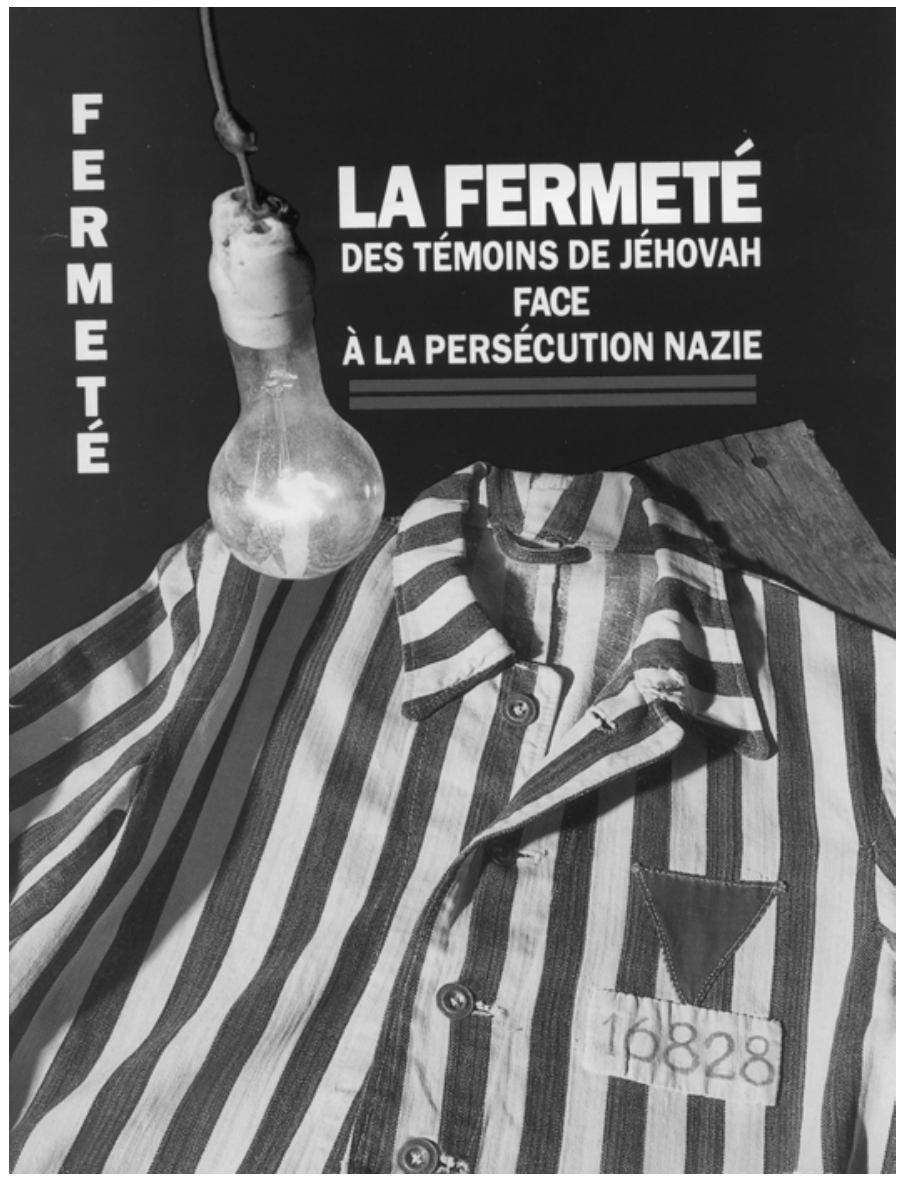

Photo et commentaire tirés d'une jaquette de cassette vidéo distribuée par les Témoins de Jéhovah, Watch Tower Bible and Tract Society of Pennsylvania, USA, 1997.

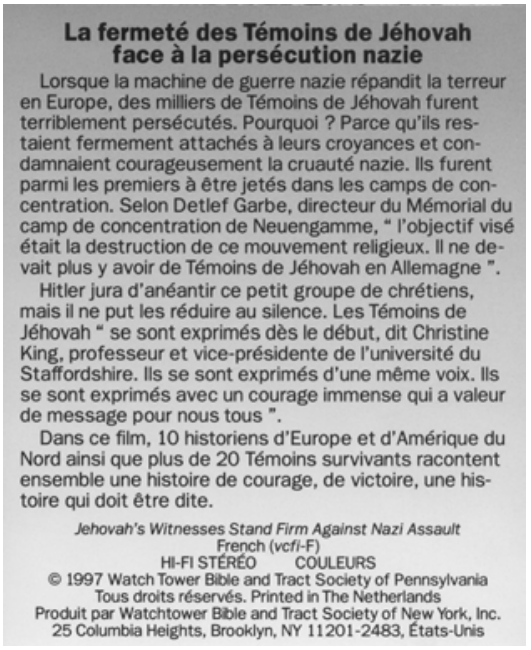




\section{Annexe 1: Souffrance et acquisition de compétences}

Pour mieux cerner le rapport entre la compétence et la souffrance, nous nous sommes intéressé à cette dernière dans l'acquisition des connaissances scolaires par le biais de l'appel à l'effort. Faire un effort consiste à en "faire plus ", c'est-à-dire à se dépasser en s'imposant un temps de travail supplémentaire pris sur le temps de loisir et de détente, à s'appliquer, c'est-à-dire à se concentrer davantage sur le travail que sur les autres activités, chose que l'élève ne fait pas toujours, peut-être en raison de ses limites personnelles et sociales. Nous avons voulu vérifier empiriquement que l'effort est lié au déplaisir et à une forme de souffrance.

L'école a pour vocation d'élever les jeunes gens à un niveau de compétence, en l'occurrence un niveau de connaissance ou de pratique (sport, musique, art) vérifiable grâce à des épreuves correspondant à une gradation dans les exercices et dans des épreuves d'évaluation standardisés (test des acquisitions scolaires).

Nous avons rassemblé un échantillon de 15 dossiers d'élèves scolarisés de la $6^{\mathrm{e}}$ à la $3^{\mathrm{e}}$ dans un collège public du nord de la France. Nous avons choisi au hasard les dossiers de cinq élèves "forts " (moyenne calculée allant de 14,70 à 17,90), cinq élèves «moyens " (moyenne calculée allant de 10,81 à 13,27) et de cinq élèves " faibles " (moyenne calculée allant de 6,93 à 9,94). Chaque dossier comporte entre sept et douze bulletins trimestriels selon la durée de la scolarité dans l'établissement.

Chaque bulletin comporte un espace par discipline portant des sous-divisions pour la moyenne de l'élève, la moyenne de la classe et l'avis de l'enseignant donné selon trois critères : "connaissances et compétence ", "progrès dans le travail et comportement " et « conseils pour progresser ». Les enseignants ne respectent pas toujours les cases et préfèrent parfois écrire leurs remarques transversalement en une phrase ou en plusieurs phrases courtes.

Sur l'ensemble des bulletins, l'appel à l'effort apparaît 308 fois (chiffre absolu donné à titre indicatif car non comparé au nombre d'autres mots selon une analyse 
lexicale), soit pour demander de poursuivre les efforts, soit pour demander d'en faire, soit pour constater que l'élève en a fait. On trouve une différence de répartition du mot " effort " puisque son occurrence est la suivante :

Bons élèves : 34 / Élèves moyens : 128 /Élèves faibles : 142.

On retrouve le même ordre de répartition du mot " effort " dans l'appréciation globale du professeur principal puisque sur l'ensemble des évaluations globales lisibles, le pourcentage des unités de sens comportant le mot s'établit ainsi :

Bons élèves : $31 \%$ / Élèves moyens : 54\% / Élèves faibles : $58 \%$.

Chez les forts, le mot "effort " est utilisé pour saluer ce dernier ou pour demander d'en faire dans une discipline où il n'excelle pas ou tout simplement pour demander de le poursuivre. L'hypothèse implicite est peut-être que l'élève réussit grâce à ses efforts plus qu'à son intelligence ou à son appartenance à un milieu social favorable.

L'appel à l'effort apparaît important. Il est un axe de jugement explicite de l'élève. Le recours à la notion d'effort est encore plus évident lorsqu'on étudie les conseils donnés par les enseignants pour parvenir à un niveau de compétence requis ou pour le maintenir. Les bulletins comportent en général deux types de conseils donnés aux élèves : d'une part des incitations à faire des efforts, à les poursuivre ou à les intensifier, d'autre part des conseils techniques. Ces derniers portent sur les modes d'apprentissage (travailler l'écrit, réciter les leçons à quelqu'un, soigner la présentation des travaux), sur des attitudes (se ressaisir, respecter la volonté de la classe de travailler, ne pas se laisser distraire, ne pas s'absenter souvent). Nous avons relevé les nombres d'appel à l'effort et de conseils techniques dans les bulletins à titre de comparaison.

\begin{tabular}{|c|c|c|c|}
\hline Niveau & appel à l'effort & conseils techniques relationnels & Total \\
\hline Forts & $240(85,10 \%)$ & $42(49,89 \%)$ & 282 \\
\hline Moyens & $151(49,83 \%)$ & $152(50,16 \%)$ & 303 \\
\hline Faibles & $180(46,87 \%)$ & $204(53,16 \%)$ & 384 \\
\hline Total & 571 & 398 & 969 \\
\hline
\end{tabular}

Il ressort de ce tableau que plus l'élève est faible, plus il reçoit de conseils. Les élèves forts sont invités à poursuivre l'effort pour maintenir leur niveau de compétence, tandis 
que les autres reçoivent plus de conseils techniques pour acquérir la compétence requise bien que la différence entre les deux types de conseils soit relativement faible; et encore, on constate que les conseils techniques recouvrent le plus souvent des appels tacites à l'effort (approfondissez le travail à la maison, concentrez-vous...) et relèvent rarement de techniques d'apprentissage. Globalement, l'appel à l'effort est important. Pour l'ensemble de l'échantillon, il est de 571 (58, $92 \%)$ contre 398 (41,07\%).

L'appel à l'effort, donc à une forme de souffrance (cf. annexe 3), est un axe majeur dans l'acquisition de connaissances.

\section{Annexe 2 : La souffrance est-elle un critère de compétence dans le travail?}

Dans un second temps, nous avons voulu savoir si la souffrance dans l'exercice d'une tâche professionnelle était reconnue comme un critère de compétence. Pour cela, nous avons proposé à 71 étudiants de psychologie (1 $1^{\text {ère }}$ et $2^{\mathrm{e}}$ année, licence, DESS de psychologie du travail) une liste de compétences appliquées à un travail. La consigne était de choisir trois critères et de les classer de 1 à 3 selon leur importance. En attribuant une note 3 au premier choix, 2 au second choix et 1 au troisième choix nous avons pu pondérer les choix. Nous obtenons donc le nombre de choix pour chaque item et une note pondérée selon le rang pour chaque item.

Les résultats sont les suivants :

\begin{tabular}{|c|c|c|c|c|}
\hline Item & $\begin{array}{c}\text { Nbre de } \\
\text { choix }\end{array}$ & $\begin{array}{c}\text { Note de } \\
\text { l'item }\end{array}$ & $\begin{array}{c}\text { Note } \\
\text { maximale }\end{array}$ & $\begin{array}{c}\text { Note } \\
\text { minimale }\end{array}$ \\
\hline $\begin{array}{l}\text { Maîtrise bien les techniques à son } \\
\text { poste de travail }\end{array}$ & 43 & 104 & 129 & 43 \\
\hline $\begin{array}{l}\text { A suivi la formation professionnelle } \\
\text { nécessaire à la réalisation de la tâche }\end{array}$ & 44 & 103 & 132 & 44 \\
\hline $\begin{array}{l}\text { Suit l'évolution des connaissances } \\
\text { dans sa profession }\end{array}$ & 55 & 97 & 165 & 55 \\
\hline Sait travailler en équipe & 30 & 55 & 90 & 30 \\
\hline $\begin{array}{l}\text { Se donne beaucoup de mal pour } \\
\text { faire de son mieux }\end{array}$ & 15 & 26 & 45 & 15 \\
\hline N'est jamais débordé par son travail & 5 & 9 & 15 & 5 \\
\hline Total & $208^{*}$ & 426 & 624 & 208 \\
\hline
\end{tabular}


On constate que le critère connotant la souffrance, "se donne beaucoup de mal ", ne figure pas parmi les items les plus choisis. Il arrive à l'avant-dernier rang avec 15 choix notés ensemble 26 (soit en dessous de la moyenne théorique qui est 30). Nous avons pensé que l'expression «se donne beaucoup de mal » était peut-être excessive et produisait un rejet. Aussi avons-nous testé la série avec l'item «fait des efforts pour faire de son mieux " au lieu du précédent mais les résultats ne différent pas sur ce point.

Interrogés sur ce point lors d'un feed-back, les étudiants du DESS de psychologie du travail nous ont dit que "c'était normal " puisque quand on sait faire le travail demandé, on ne se donne pas de mal.

Nous constatons donc que le critère "effort " - une forme de souffrance - est présent dans l'acquisition des compétences mais qu'une fois celles-ci acquises, la souffrance en est exclue. Les aspects techniques (savoir et savoirfaire) sont privilégiés dans l'évaluation.

\section{Annexe 3: L'effort est-il associé à la souffrance?}

La réponse à cette question est un préalable à la première étude.

Pour savoir si l'effort réclamé aux élèves par les enseignants est associé à la souffrance, nous avons proposé une épreuve d'associations de mots à partir du mot inducteur " effort " à des étudiants en Administration économique et sociale et à la classe d'élèves qui a fourni notre échantillon de dossiers scolaires. Chez les étudiants, nous avons demandé dix mots et nous avons retenu les trois premiers, chez les élèves, l'enseignant a demandé cinq mots, tous retenus dans le traitement. Chez les étudiants nous avons disposé de 29 listes soit 87 termes que nous avons regroupés en dix catégories. 


\begin{tabular}{|c|c|c|c|}
\hline Catégorie & $\begin{array}{c}\text { Nbre } \\
\text { de } \\
\text { mots }\end{array}$ & $\%$ & connotation \\
\hline Difficulté (difficile, difficulté, se forcer) & 16 & 18,39 & négative \\
\hline Travail (travail, labeur, études) & 18 & 16,00 & \\
\hline $\begin{array}{c}\text { Sensations désagréables (sueur, peine épuise- } \\
\text { ment, douleur) }\end{array}$ & 17 & 19,54 & négative \\
\hline $\begin{array}{c}\text { Aptitudes associées (courage, concentration, } \\
\text { résistance, capacité, rigueur, motivation...) }\end{array}$ & 19 & 21,83 & \\
\hline Résultats positifs (progresser...) & 5 & 5,74 & \\
\hline Sensations agréables & 2 & 2,29 & positive \\
\hline But à atteindre & 2 & 2,29 & positive \\
\hline La contrainte & 3 & 3,44 & négative \\
\hline Mots positifs (espoir, utile) & 2 & 2,29 & positive \\
\hline Hors catégorie (sport, soleil..) & 5 & 5,74 & \\
\hline Total & 87 & 99,93 & \\
\hline
\end{tabular}

La somme des pourcentages de catégories négatives $(41,37 \%)$ est nettement supérieure à celle des catégories positives (10,32\%). Chez les étudiants l'effort semble connoté négativement. Il se situe plus du côté de la contrainte, des sensations désagréables que du côté des sensations agréables. Il se situe à l'opposé du bien-être et, de ce fait, on peut le rapprocher de la souffrance.

Chez les élèves de troisième de collège, nous avons obtenu 25 listes formant un total de 110 citations, certaines listes étant incomplètes.

Comme précédemment, nous avons procédé à un regroupement en 10 catégories.

\begin{tabular}{|c|c|c|c|}
\hline Catégories & $\begin{array}{c}\text { Nbre de cita- } \\
\text { tions }\end{array}$ & $\%$ & connotation \\
\hline Mots exprimant la difficulté & 13 & 11,83 & Négative \\
\hline Mots exprimant le travail & 19 & 17,27 & \\
\hline Sensations désagréables & 20 & 18,08 & Négative \\
\hline Vocabulaire du sport & 32 & 29,09 & \\
\hline Aptitudes associées & 17 & 15,45 & \\
\hline Mots exprimant le but & 1 & 0,90 & \\
\hline Sensations agréables & 1 & 0,90 & Positive \\
\hline Résultats positifs de l'effort & 4 & 3,63 & Positive \\
\hline Mots sans statut & 3 & 2,72 & \\
\hline Total & 110 & 99,97 & \\
\hline
\end{tabular}

Les élèves de collège ont privilégié le vocabulaire du sport, qui n'est pas présent chez les étudiants. Cela tient 
sans doute aux préoccupations de l'âge. Toutefois, la distinction entre les aspects négatifs de l'effort et les aspects positifs de celui-ci apparaissent encore : $29,99 \%$ des mots associés à l'effort le situent du côté négatif contre 4,53\% qui le situent du côté positif. Les mots "souffrance" et «torture " apparaissent même parmi les associations.

Il ne nous paraît pas utile d'étendre ce test à un échantillon plus grand à cause du caractère répétitif des inductions d'un mot. La redondance est importante car le stock de mots induits par un autre mot est limité. Nous pensons avoir montré grâce à ce test la présence d'un lien entre l'effort, la pénibilité, le "forçage ", qui nous semble proche d'une définition large de la souffrance.

\section{Annexe 4: Les critères d'une vraie religion (compétence religieuse)}

Pour tester le rôle de la souffrance dans la reconnaissance d'une compétence religieuse, nous avons proposé à 101 étudiants une liste de critères de ce qui pourrait être une "véritable religion ". Nous avons choisi cette expression pour éviter celle de compétence religieuse qui n'évoquerait peut-être rien dans l'esprit des personnes interrogées. Cette expression a pour avantage de renvoyer au vocabulaire que les médias véhiculent - «fausse religion» opposée à la "vraie religion»- en reprenant les termes des opposants aux sectes chrétiennes.

Parmi les critères proposés, certains se rapportent à la souffrance (les martyrs, le soutien psychologique aux personnes en détresse, le fait de surmonter les épreuves de la vie, la persécution). D'autres portent sur les aspects tangibles (clergé, cérémonies, lieu de culte, sacralisation des passages de la vie). D'autres encore sur les buts classiques de la religion (connaissance de Dieu, perfection morale, sens de l'existence, conversion). D'autres enfin renvoient aux engagements dans le monde: défense des droits de l'homme, protestation sociale, activités humanitaires. Deux items étaient atypiques ("liberté laissée aux fidèles de ne pas croire en Dieu » et " développement des capacités "). 
Les étudiants avaient pour consigne de choisir trois critères caractérisant un mouvement spirituel comme véritable religion et de les numéroter de 1 à 3 par ordre d'importance. Une note de 3 pour le premier choix, 2 pour le second choix et 1 pour le troisième choix permet de pondérer les choix (note de l'item).

Un mouvement spirituel est une véritable religion si :

\begin{tabular}{|c|r|r|}
\hline Item & $\begin{array}{c}\text { Nbre } \\
\text { de } \\
\text { choix }\end{array}$ & Note \\
\hline Il a des activités humanitaires & 13 & 23 \\
\hline Il a eu des martyrs à cause de ses croyances & 1 & 1 \\
\hline Il soutient psychologiquement les personnes en détresse & 20 & 52 \\
\hline $\begin{array}{c}\text { Il proteste contre certaines pratiques sociales (corruption, } \\
\text { injustices sociales, société de consommation) }\end{array}$ & 8 & 14 \\
\hline Il défend les droits de l'homme & 28 & 66 \\
\hline Il demande aux hommes de se perfectionner moralement & 29 & 57 \\
\hline Il permet de connaitre Dieu & 21 & 48 \\
\hline Il permet à ses fidèles de surmonter les épreuves de la vie & 41 & 94 \\
\hline Il a un clergé & 2 & 5 \\
\hline Il s'efforce de convertir les hommes & 6 & 10 \\
\hline Il a des lieux pour se réunir et prier & 20 & 28 \\
\hline Il a des cérémonies religieuses & 8 & 11 \\
\hline Il aide les hommes à développer leurs capacités. & 9 & 16 \\
\hline Il sacralise les moments importants de la vie (naissance, \\
adolescence, mariage, décès) & 20 & 30 \\
\hline Il a été persécuté (ou il l'est encore) & 1 & 1 \\
\hline Il laisse à ses fidèles la possibilité de ne pas croire en Dieu & 33 & 97 \\
\hline Il donne un sens à la vie & 43 & 94 \\
\hline
\end{tabular}

On note que la dispersion des choix est grande à cause du nombre de propositions.

Parmi les principaux critères choisis par les étudiants pour qualifier une "vraie religion", c'est-à-dire pour attribuer une compétence religieuse, on trouve par ordre: "donner un sens à la vie " (43 choix), " permet à ses fidèles de surmonter les épreuves de la vie " (41 choix), « laisse à ses fidèles la possibilité de ne pas croire en Dieu " (33 choix). La souffrance endurée par les fidèles et les martyrs viennent en dernier avec 1 choix chacun.

Les items les mieux notés sont par ordre : la possibilité laissée aux fidèles de ne pas croire en Dieu (97), puis exaequo : «permet à ses fidèles de surmonter les épreuves de 
la vie " et "donne un sens à la vie " (94). Beaucoup plus loin derrière arrivent la défense des droits de l'homme (66), le perfectionnement moral (57) et le soutien psychologique (52). La persécution et le martyre n'obtiennent qu'un point chacun.

Naturellement, il ne s'agit pas d'un cliché obtenu dans une population globale représentative des Français, mais d'un coup de sonde. Néanmoins, dans notre population étudiante, on aperçoit une tendance actuelle : l'image qui se dessine derrière les critères de "vraie religion " est plutôt celle de religions asiatiques, de groupes du Nouvel Âge et de religions de guérison : religion sans Dieu avec une voie de la libération des souffrances et une cosmogonie qui donne un sens à la vie. Quoi qu'il en soit, nous avons montré l'importance accordée au dépassement des souffrances et à l'aide psychologique (facteurs de résilience). La souffrance seule n'est pas considérée comme un critère de compétence.

\section{Annexe 5: La notion de culte reconnu et l'évaluation d'un mouvement religieux}

Nous avons soumis deux questions suivies d'une courte introduction à une population de 64 étudiants de l'UFR IDIST (documentation) et de psychologie.

En France, l'Etat a le pouvoir de donner à une religion le statut de «culte reconnu ». Ce statut procure des avantages fiscaux et le droit de recevoir des dons et des héritages. Tous les mouvements religieux n'en bénéficient pas.

1) Si vous aviez à donner votre opinion sur un groupe religieux, le fait qu'il soit reconnu par l'Etat aurait-il un effet positif sur celle-ci? Oui/Non/NSP.

2) Si oui, la reconnaissance par l'Etat signifie-t-elle pour vous que nous avons affaire à une vraie religion? Oui/Non/NSP.

\section{Résultats}

\begin{tabular}{|c|c|c|c|c|}
\hline Première question & Oui $: 17$ & Non $: 43$ & NSP $: 4$ & $T=64$ \\
\hline Deuxième question & Oui $: 4$ & Non $: 12$ & NSP $: 1$ & $T=17$ \\
\hline
\end{tabular}




\section{Conclusions}

- Le fait d'être un culte reconnu ne contribue pas majoritairement à un avis favorable.

- Dans le cas où cela contribue à un effet favorable, la reconnaissance n'est pas un certificat de « vraie religion " (manière d'opérationaliser une compétence religieuse).

\section{Annexe 6}

Surpris par les résultats précédents, nous avons voulu vérifier une seconde fois l'importance de divers critères de compétence en proposant un questionnaire à 70 étudiants de psychologie de tous niveaux.

Cette fois nous avons demandé aux sujets de noter leur accord avec des propositions en se situant sur une échelle visualisant la notation de 1 à 7 de chaque item sur lequel ils étaient invités à se situer :

Il était précisé que 1 correspond à « désaccord total » et 7 à accord total et qu'ils pouvaient utiliser la gamme de notation intermédiaire pour donner leur avis.

Chaque note moyenne de l'item a été comparée à la moyenne théorique 3,5 grâce à un test de comparaison de moyennes ( $t$ de student).

Nous avons proposé 19 items que nous ne traiterons pas tous car certains n'apportent rien à notre étude. Pour éviter un effet de contamination positif entre les réponses et déceler les réponses désinvoltes nous avons inséré des items qui reçoivent une opinion négative (test préalable) tels que : "les études et les diplômes ne servent à rien ». Les résultats obtenus sont conformes à ceux du pré-test.

Les items qui reçoivent une note moyenne significative supérieure à la moyenne sont :

«Les groupes qui ont souffert et qui ont surmonté leur souffrances ont acquis une compétence particulière dans la vie " $(\mathrm{m}=3,74)$

"Une personne qui a souffert a acquis une meilleure connaissance de la vie que celle qui n'a pas souffert " $(\mathrm{m}=3,98)$

«Un travail qui a été fait dans l'effort doit être mieux apprécié subjectivement qu'un travail fait avec facilité même si les deux aboutissent au même résultat » $(m=4,57)$ 
52 Je remercie Claude Lemoine, professeur de psychologie sociale et du travail pour son dialogue à propos de l'article.
«On peut être harcelé moralement à cause de sa compétence dans un domaine " $(\mathrm{m}=5,2)$

On trouve que l'item «L'acquisition de compétences s'accompagne souvent d'une certaine souffrance " reçoit une note de 3,5, soit la moyenne théorique. En revanche l'item « Un groupe de croyants ne devient une vraie religion que s'il a été persécuté dans ses débuts " reçoit une note négative ( $m=2,07$, significatif).

Litem «Les Témoins de Jéhovah ont été persécutés sous le régime nazi et dans les pays totalitaires. Ils ont survécu à leur souffrances en s'appuyant sur leurs croyances. Pour cela, on peut leur accorder une compétence religieuse même si on n'est pas d'accord avec eux " reçoit une moyenne de 3,24 (non-significative), donc du même ordre que la moyenne théorique.

Les items liant les souffrances de groupes particuliers (Juifs, Protestants, Palestiniens) à des compétences similaires (meilleure connaissance de la vie, de la société, meilleure connaissance des hommes) sont négativement jugés (significatifs) alors que l'item général "Les groupes qui ont souffert ont acquis une compétence particulière dans la vie " reçoit une note significativement supérieure à la moyenne. Le paradoxe est à expliquer. Les compétences attribuées étaient-elle inadéquates? Les souffrances de ces groupes évoquent-ils l'indignation plus que de l'attribution de compétence. Un autre étude serait à faire pour expliquer le paradoxe.

De la même façon, le score obtenu par l'item «On peut être harcelé moralement à cause de sa compétence dans un domaine" est surprenant. Un groupe religieux peut l'utiliser comme le font les Témoins selon la formule " celui qui dit la vérité sera persécuté ", mais l'accord avec l'item peut relever de la projection d'un sentiment de jalousie dans une situation scolaire.

Pour nous, il suffit de constater que la souffrance est liée à la compétence mais plus encore à la souffrance surmontée. Ici encore les résultats confirment un hiatus entre l'évaluation générale de la compétence par le fait de surmonter la souffrance et l'évaluation de son application aux témoins de Jéhovah ${ }^{52}$. 


\section{Références bibliographiques}

Arnaud, M.

2003 La résilience. Surmonter les traumatismes, Paris, Nathan.

Bettheleim, B.

1972 Le cour conscient, Paris, Robert Laffont.

Botting, G.

1993 Fundamentals freedoms and Jehovah's Witnesses, Calgary, University of Calgary Press.

Canonici, G.

1998 Les Témoins de Jéhovah face à Hitler, Paris, Albin Michel, Préface de F. Bedarida.

Courpasson, D. \& Y.F. Livian

1991 "Le développement récent de la notion de "compétence" ", Revue de gestion des ressources humaines 1, pp. 3-10.

Cyrulnik, B.

1999 Un merveilleux malheur, Paris, Odile Jacob.

Déjours, C.

1998 La souffrance en France, Paris, Seuil.

Delestre, A.

1977 Les religions des étudiants, Paris, L'Harmattan.

Desrumaux, P.

1996 Explications causales et engagement contre ou pro-attitudinal. De l'internalité aux conduites pro-attitudinales, Thèse de l'Université Charles de Gaulle-Lille.

Detraux, J.J.

2002 «De la résilience à la bientraitance de l'enfant handicapé et de sa famille; essai d'articulation de divers concepts ", Pratiques psychologiques 1, pp. 29-40.

Gannon,T. M.

1971 "Priest/Minister : Profession or Not Profession?", Review of Religious Research, pp. 66-79.

Houssier, F.

2002 "Clinique du traumatisme et résilience : regards sur le parcours de B. Betthelheim ", Pratiques psychologiques 1, pp. 65-73.

King, C.E.

1982 "The Case of the Third Reich ", in Eileen Barker (dir.), New religious movements : a perspective for understanding Society, New York \& Toronto, The Edwin Mellen Press, pp. 125-139. 
Les Témoins de Jéhovah

1939 Fascisme ou liberté, Brooklyn, Watchtower Bible and Tract Society.

1971 [1959 pour l'édition anglaise] Les Témoins de Jéhovah dans les desseins divins, Brooklyn, Watchtower Bible and Tract Society.

1993 Les Témoins de Jéhovah, prédicateurs du Royaume de Dieu, Brooklyn, Watchtower Bible and Tract Society.

Levy-Leboyer, C.

2004 [1996] La gestion des compétences, Paris, éditions de l'organisation.

Montmollin (de), M.

1993 "L'ergonome, le pilote et la femme du directeur", in J. d'Aubret, P. Gilbert et F. Pigeyre (dir.), Savoir et pouvoir, les compétences en question, Paris, P.U.F., pp. 205-210.

2001 «La compétence », in J. Leplat et M. de Montmollin (dir.), Les compétences en ergonomie, Toulouse, Octares, pp. 11-12.

Nowak, E.

1972 Le Chrétien devant la souffrance, étude sur la pensée de Jean Chrysostome, Paris, Beauchesne.

Pelchat, D., H. Lefebvre \& C. Damiani

2002 «Deuil, appropriation de compétences. Transformation." Pratiques Psychologiques 1, pp . 41-52.

Penton, J.

1976 Jehovah's Witnesses in Canada, Toronto, Macmillan.

Séguy, J.

1982 «Le clergé dans une perspective sociologique. Que faisons-nous de nos classiques? » in Prêtres, pasteurs et rabbins dans la société contemporaine, Actes $\mathrm{du} \mathrm{VI}^{\mathrm{e}}$ colloque de sociologie du protestantisme, Paris, Cerf, pp.11-85.

Weber, M.

1964 Ethique protestante et esprit du capitalisme, Paris, Plon.

1996 Sociologie de la religion, Paris, Gallimard.

2003 [1921] Hindouisme et Bouddhisme, Paris, Flammarion.

Weiner \& Kukla

1970 "An attributional analysis of achievement motivation", Journal of Personality and Social Psychology 15, 1, 1-20.

Willaime, J.P.

1989 Profession Pasteur, Genève, Labor et Fides.

Zuber, V. \& J. Baubérot

2000 Une haine oubliée, l'antiprotestantisme avant le "pacte laïque "(1870-1905),

Paris, Albin Michel. 